Sitzungsberichte

der Akademie der Wissenschaften der DDR

Mathematik - Naturwissenschaften - Technik

1977

\title{
Rudolf Engst
}

Zum Reaktionsverhalten

ausgewählter

Fremdstoffe 
